\title{
Morcegos (Chiroptera) da área urbana de Londrina, Paraná, Brasil
}

\author{
Nélio Roberto dos Reis ${ }^{1}$ \\ Isaac Passos de Lima ${ }^{2}$ \\ Adriano Lúcio Peracchi ${ }^{3}$
}

\begin{abstract}
Bats (Chiroptera) of the urban area of Londrina, Paraná, Brazil. Study carried out within the urban perimeter of Londrina, which is located in the North of the state of Paraná. The objectives were the identification of urban species of bats and diurnal roosts used by them and the verification of the problems they can cause to the population. The fire brigade, the Autarquia Municipal do Ambiente de Londrina (Municipal Environment Autarchy of Londrina), the Biology Department of the Universidade Estadual de Londrina (State University of Londrina) and local residents helped spot the roosts. The collections were carried out in regular intervals between April 1998 and March 1999. By the end of them, 815 bats of 23 different species had been captured. Among these, 12 were found near or inside human constructions: Noctilio albiventris Desmarest, 1818; Artibeus lituratus (Olfers, 1818); Platyrrhinus lineatus (E. Geoffroy, 1810); Eptesicus brasiliensis Desmarest 1819; Lasiurus borealis (Muller 1776); Lasiurus ega (Gervais, 1856); Eumops glaucinus (Wagner, 1843); Molossus rufus (E. Geoffroy, 1805); Molossus molossus (Pallas, 1766); Nyctinomops laticaudatus (E. Geoffroy, 1805); Nyctinomops macrotis (Gray, 1840) e Tadarida brasiliensis (I. Geoffroy, 1824). Roost sites comprised expansion joints, roofs, attics and parks, among others. It can be concluded that bats are treated as undesirable animals by the population due to the lack of knowledge about the subject.

KEY WORDS. Chiroptera, Bats, urban bats
\end{abstract}

Foi constatada em 1997, pela $17^{\text {a }}$ Regional de Saúde do Estado do Paraná, cuja sede fica em Londrina, a presença do vírus da raiva em um exemplar de Molossus sp. capturado na área urbana desse município, o que trouxe preocupação aos órgãos competentes.

Essa preocupação se justifica em razão da diversidade de espécies de morcegos existente na região de Londrina. Trabalhos realizados em suas principais unidades de conservação, como o Parque Estadual Mata dos Godoy (REIS et al. 1993a, b; REIS \& MULlER 1995) e o Parque Municipal Arthur Thomas, e em outras áreas verdes, como os fundos de vales (REIS et al. 1998, 2000), já detectaram a presença de 41 espécies de morcegos.

1) Departamento de Biologia Animal e Vegetal, Universidade Estadual de Londrina. 86051-990 Londrina, Paraná, Brasil.

2) Pós-Graduação em Ciências Biológicas, Universidade Estadual de Londrina. 86051-990 Londrina, Paraná, Brasil.

3) Departamento de Zoologia, Instituto de Biologia, Universidade Federal Rural do Rio de Janeiro. 23851-970 Seropédica, Rio de Janeiro, Brasil. 
Essa diversidade de morcegos em ambientes florestais da região, e o fato de que mesmo aquelas espécies não hematófagas são potenciais transmissoras do vírus da raiva (UIEDA 1998; B AER 1991; ALMEIDA et al. 1994; UIEDA et al. 1996; SILVA et al. 1996a, 1999), aliados à inexistência de dados sobre quais espécies estão presentes no ambiente urbano londrinense, trouxe a necessidade de se realizar um estudo que, num primeiro momento, identificasse as espécies e os locais de repouso que utilizam. A identificação das espécies e um melhor conhecimento de sua biologia poderiam auxiliar na resolução de problemas relacionados a esses animais.

Assim, este estudo teve como objetivos identificar as espécies presentes no perímetro urbano de Londrina, determinar quais os tipos de abrigo mais utilizados pelos morcegos em ambientes urbanos e verificar as principais implicações de sua presença nesses ambientes.

Londrina surgiu no início da década de $30 \mathrm{e}$, devido à fertilidade de seu solo (do tipo Latossolo), atraiu muitos colonizadores de várias regiões do país, principalmente para o cultivo de café, o que reduziu em muito seu ecossistema original. Sua área urbana ocupa $99.312 .322 \mathrm{~m}^{2}$, abrigando uma população de 424.573 habitantes; e sua área verde é de $7.711 .227,3 \mathrm{~m}^{2}\left(22,2 \mathrm{~m}^{2}\right.$ /habitante), havendo 200 praças públicas (IBGE 2000). O que resta, hoje, de seu ecossistema original, são pequenos remanescentes formando ilhas de matas circundadas por áreas de intensa exploração agrícola e, destes, alguns foram transformados em unidades de conservação.

A área metropolitana de Londrina, no norte de Paraná (Fig. 1), está localizada nas coordenadas geográficas $23^{\circ} 14^{\prime}-23^{\circ} 23^{\prime} \mathrm{S}$ e $51^{\circ} 05^{\prime}-51^{\circ} 14^{\prime} \mathrm{W}$. O município tem altitude média de $700 \mathrm{~m}$ e clima subtropical úmido com chuvas em todas as estações, podendo ocorrer secas no inverno. A temperatura média máxima para o verão é $39^{\circ} \mathrm{C}$, e a média mínima para o inverno é $10,4^{\circ} \mathrm{C}$; a pluviometria média é $1.615 \mathrm{~mm}$, sendo os meses mais chuvosos os de dezembro e janeiro, e o de menor pluviosidade o de agosto ReIS et al. (1998).

Com relação à vegetação urbana, a área de maior relevância para a cidade é o Parque Municipal Arthur Thomas, que, no entanto, não possui características urbanas. Outras áreas, de menor tamanho, são o horto florestal da Universidade Estadual de Londrina (UEL) (aproximadamente 10 ha da área total do campus universitário, que compreende 150 ha), a mata do ribeirão Cambezinho, algumas matas de galeria e de nascentes de cursos hídricos e, ainda, o bosque central de Londrina (praça Marechal Cândido Rondon).

Em alguns fundos de vales e praças londrinenses podem ser encontradas plantas introduzidas, como a bisnagueira, Spathodea campanulata Beauv. - (Bignoniaceae), a cigarreira, Cassia sp. (Leguminosae, Caesalpinoideae) e a sibipiruna, Caesalpinia sp. (Leguminosae, Caesalpinoideae); plantas ornamentais como o hibisco, Hibiscus sp. (Malvaceae), o lírio branco, Hippeastrum sp. (Hemerocallidaceae) e o lírio amarelo, Hemerocallis sp. (Amaryllidaceae); e árvores frutíferas como goiaba (Psidium guajava L., Myrtaceae), amora (Morus nigra L., Moraceae) e sete-copas (Terminalia catappa L., Combretaceae) - a nomenclatura das famílias está de acordo com BRUMMITT (1992). Essa vegetação contribui para o fornecimento de alimento e abrigo a várias espécies de animais. 
Os fundos de vales encontram-se degradados em quase toda sua extensão urbana, sem a presença de mata ciliar e, quando esta aparece, é insuficiente para proteger o curso hídrico, quando presente, e gerar alimento à fauna aquática. São usados como depósito de lixo residencial, industrial e da construção civil, além de serem alterados por aterros e instalação de favelas (SEPLAN 1993).

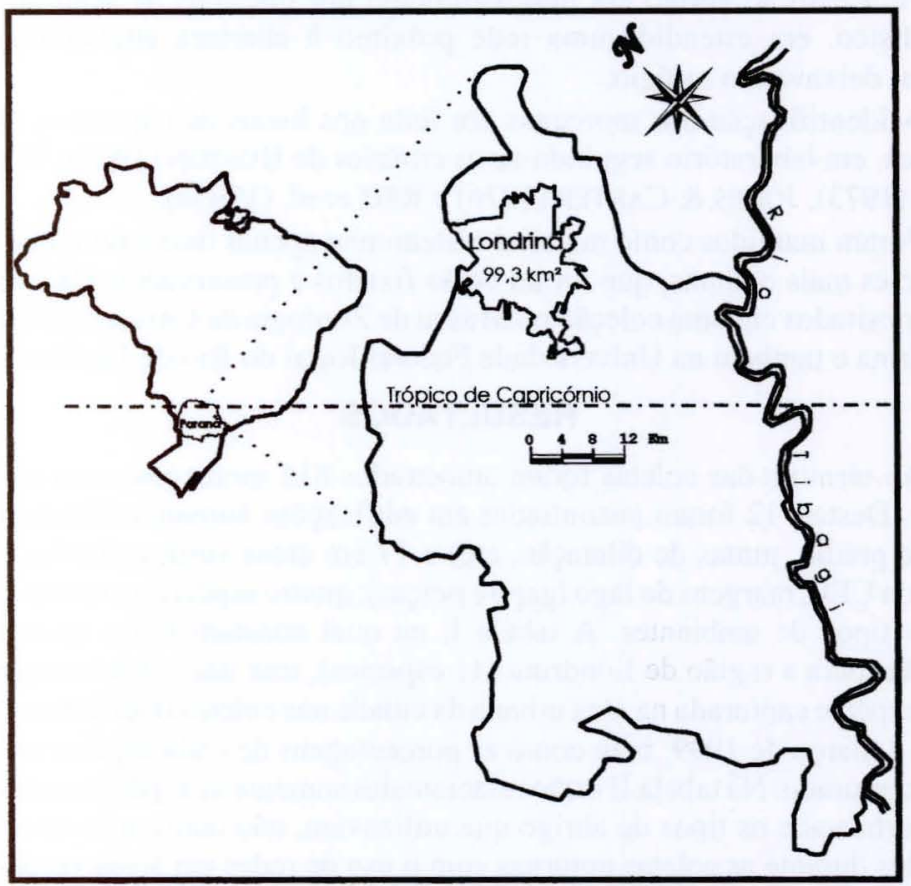

Fig.1. Localização da cidade de Londrina, Paraná.

\section{MATERIAL E MÉTODOS}

Foram realizadas nove coletas (três noturnas e seis diurnas) em cada estação do ano, totalizando 36 coletas entre abril de 1998 e março 1999. As coletas noturnas foram adaptadas de GREENHALL \& PARADISO (1968), realizadas em noites com ausência de lua, em praças pouco movimentadas e fora dos locais com muita iluminação artificial, e também em ilhas de matas que restaram no perímetro urbano, nos fundos de vales, nas margens do lago Igapó e no campus da UEL. Nas coletas noturnas, redes-neblina (mist nets) permaneceram estendidas nas primeiras três horas após o pôr-do-sol, sendo totalizados, para cada local de coleta, $90 \mathrm{~m}^{2}$ de redes. Nas coletas diurnas, feitas de acordo com BREDT et al. (1996), eram visitados locais como forros de casas, forros de prédio, juntas de dilatação, bueiros e fendas de pedras, entre outros. Para a localização desses abrigos houve ajuda do Corpo de Bombeiros e da Autarquia Municipal do Ambiente de Londrina (AMA); outros abrigos foram localizados a partir de denúncias feitas junto ao Departamento de 
Biologia da UEL e por indicações de pessoas do convívio próximo e, ainda, mensagens eletrônicas foram enviadas para vários usuários da internet na cidade, solicitando informações.

Para a captura dos animais encontrados em forros eram utilizadas luvas de couro, e eles eram então colocados em sacos de algodão, para posterior identificação; quando o acesso ao abrigo era impossibilitado por questões de segurança ou de espaço físico, era estendida uma rede próximo à abertura através da qual os morcegos deixavam o refúgio.

A identificação dos morcegos era feita nos locais de captura e, nos casos duvidosos, em laboratório seguindo-se os critérios de HuSSON (1962), VizotTO \& TADDEI (1973), JONES \& CARTER (1976) e REIS et al. (1993a).

Foram mantidos como material-testemunho apenas dois exemplares daquelas espécies mais comuns, que foram então fixados e preservados. Os exemplares estão depositados em uma coleção no Museu de Zoologia da Universidade Estadual de Londrina e também na Universidade Federal Rural do Rio de Janeiro.

\section{RESULTADOS}

Ao término das coletas foram amostrados 815 morcegos, num total de 23 espécies. Destas, 12 foram encontradas em edificações humanas (forros de casas, forros de prédio, juntas de dilatação, etc) e 17 em áreas verdes (fundos de vales, campus da UEL, margens do lago Igapó e praças); quatro espécies estavam presentes nos dois tipos de ambientes. A tabela I, na qual constam todas as espécies já registradas para a região de Londrina (41 espécies), traz nas colunas a quantidade de cada espécie capturada na área urbana da cidade nas coletas realizadas entre abril de 1998 e março de 1999, bem como as porcentagens de cada espécie em relação ao total capturado. Na tabela II estão relacionadas somente as espécies coletadas em abrigos urbanos e os tipos de abrigo que utilizavam, não constando os espécimes capturados durante as coletas noturnas com o uso de redes em áreas verdes.

\section{DISCUSSÃO}

Das 41 espécies já identificadas na região de Londrina, 23 foram encontradas no perímetro urbano, sendo que 12 ao redor de edificações humanas, convivendo diretamente com o homem. A destruição dos habitats naturais dos morcegos criou uma realidade onde esses animais passaram a conviver de forma mais intensiva com os humanos. Em ambientes urbanos, morcegos são tratados como animais indesejáveis. Além do medo que as pessoas têm desses animais, contribui para essa animosidade o fato de eles atacarem pomares domésticos para se alimentar, o acúmulo de suas fezes nos forros das casas (muitas vezes, as fezes caem no interior das casas), o odor forte de sua urina nos dias de calor intenso, o barulho que causam quando vocalizam e a possibilidade de transmitirem doenças como a raiva (PEDRO 1998), como qualquer outro mamífero doméstico.

Os morcegos insetívoros, que antes se abrigavam em ocos de árvores nas matas, agora encontram nas cidades abrigo seguro e farta alimentação de insetos, que são atraídos pelas luzes (RYDELL 1992; SiLVA et al. 1996b). Os principais abrigos que utilizam em ambientes urbanos são forros de casas, forros de prédios, tubulações 
Tabela I. Relação das espécies já registradas para a região de Londrina, e espécies registradas na área urbana desse município.

\begin{tabular}{|c|c|c|c|c|c|}
\hline \multirow{2}{*}{ Espécies } & \multicolumn{2}{|c|}{ Áreas verdes } & \multicolumn{2}{|c|}{ Edificaçōes } & \multirow{2}{*}{ Fontes ${ }^{*}$} \\
\hline & $\mathrm{N}$ & $\%$ & $\mathrm{~N}$ & $\%$ & \\
\hline \multicolumn{6}{|l|}{ Noctilionidae (1 gênero, 2 espécies) } \\
\hline Noctilio albiventris Desmarest, 1818 & & & & & 1 \\
\hline Noctilio leporinus (Linnaeus, 1758) & & & 8 & 1,4 & 6 \\
\hline \multicolumn{6}{|l|}{ Phyllostomidae (16 gêneros, 21 espécies) } \\
\hline Chrotopterus auritus (Peters, 1856) & & & & & $2,3,4$ \\
\hline Micronycteris megalotis (Gray, 1842) & & & & & $2,3,4,5$ \\
\hline Phyllostomus discolor Wagner, 1843 & 3 & 1,1 & & & 6 \\
\hline Phyllostomus hastatus (Pallas, 1767) & & & & & $3,4,5$ \\
\hline Anoura caudifer (E. Geoffroy, 1818) & & & & & 3,5 \\
\hline Glossophaga soricina (Pallas, 1766) & & & & & 3,4 \\
\hline Carollia perspicillata (Linnaeus. 1758) & 25 & 9,6 & & & $2,3,4,5,6$ \\
\hline Artibeus fimbriatus Gray, 1838 & 6 & 2,3 & & & $1,2,6$ \\
\hline Artibeus jamaicensis Leach, 1821 & & & & & $2,3,4,5$ \\
\hline Artibeus lituratus (Olfers, 1818) & 167 & 64,0 & 7 & 1,3 & $3,4,5,6$ \\
\hline Artibeus obscurus Schinz, 1821 & & & & & 7 \\
\hline Chiroderma doriae Thomas, 1891 & & & & & $2,3,5$ \\
\hline Chiroderma villosum Peters, 1860 & 1 & 0,4 & & & 1,6 \\
\hline Platyrrhinus lineatus (E. Geoffroy, 1810) & 25 & 9,6 & 1 & 0,2 & $2,3,4,5,6$ \\
\hline Pygoderma bilabiatum (Wagner, 1843) & 1 & 0,4 & & & $3,4,5,6$ \\
\hline Sturnira lilium (E. Geoffroy, 1810) & 5 & 1,9 & & & $2,3,4,5,6$ \\
\hline Uroderma bilobatum Peters, 1866 & & & & & 1 \\
\hline Vampyressa pusilla (Wagner, 1843) & & & & & $2,3,5$ \\
\hline Desmodus rotundus (E. Geoffroy, 1810) & & & & & $2,3,4,5$ \\
\hline Diaemus youngi (Jentink, 1893) & & & & & 7 \\
\hline Diphylla ecaudata (Spix, 1823) & & & & & 1 \\
\hline \multicolumn{6}{|l|}{ Vespertilionidae (5 gêneros, 11 espécies) } \\
\hline Eptesicus brasiliensis (Desmarest, 1819) & 12 & 4,6 & 14 & 2,5 & $3,4,6$ \\
\hline Eptesicus diminutus Osgood, 1915 & 1 & 0,4 & & & $3,5,6$ \\
\hline Eptesicus furinalis (d'Orbigny, 1847) & 2 & 0,8 & & & $2,3,5,6$ \\
\hline Eptesicus sp. & 2 & 0,8 & & & 6 \\
\hline Histiotus velatus (I. Geoffroy, 1824) & & & & & 3,5 \\
\hline Lasiurus borealis (Muller, 1776) & 1 & 0,4 & 1 & 0,2 & $2,3,5,6$ \\
\hline Lasiurus ega (Gervais, 1856) & & & 2 & 0,4 & 1,6 \\
\hline Myotis levis (I. Geoffroy, 1824) & & & & & 3 \\
\hline Myotis nigricans (Schinz, 1821) & 1 & 0,4 & & & $2,3,5,6$ \\
\hline Myotis ruber (E. Geoffroy, 1806) & & & & & $3,4,5$ \\
\hline Rogheessa tumida H. Allen, 1866 & & & & & 1 \\
\hline \multicolumn{6}{|l|}{ Molossidae (5 gêneros, 7 espécies) } \\
\hline Eumops glaucinus (Wagner, 1843) & & & 20 & 3,6 & 1,6 \\
\hline Molossops abrasus (Temminck, 1827) & 7 & 2,7 & & & $3,4,6$ \\
\hline Molossus rufus E. Geoffroy, 1805 & & & 214 & 38,6 & $3,4,6$ \\
\hline Molossus molossus (Pallas, 1766) & 1 & 0,4 & 38 & 6.9 & $3,4,6$ \\
\hline Nyctinomops laticaudatus (E. Geoffroy, 1805) & & & 201 & 36,3 & $3,4,6$ \\
\hline Nyctinomops macrotis (Gray, 1840) & & & 47 & 8,5 & 1,6 \\
\hline Tadarida brasiliensis (I. Geoffroy, 1824) & 1 & 0.4 & 1 & 0,2 & 3,6 \\
\hline Total (27 gêneros, 41 espécies) & 17 & 100,0 & 12 & 100,0 & \\
\hline Total de individuos & 261 & & 554 & & \\
\hline
\end{tabular}

(1) Reis et al. (1998), (2) ReIs et al. (2000), (3) ReIs et al. (1993a), (4) ReIS et al. (1993b), (5) REIS \& MULLER (1995), (6) registradas neste estudo; (7) dados não publicados. 
fluviais e juntas de dilatação das construções (Tab. II). Morcegos frugívoros, que apresentam maior potencial adaptativo, como $A$. lituratus e $P$. lineatus, passaram a se alimentar de árvores ornamentais e de frutíferas de quintal como mamão (Carica papaya L., Caricaceae), caqui (Diospyros kaki L.f., Ebenaceae), abacate (Persea americana Mill., Lauraceae), manga (Mangifera indica L., Anacardiaceae), amora (Morus nigra L., Moraceae), goiaba (Psidium guajava L., Myrtaceae), jabuticaba (Myrciaria cauliflora (Mart.) Berg, Myrtaceae), e ameixa (Eriobotrya japonica (Thumb.) Lindl., Rosaceae). Esses morcegos têm, além da frugivoria, a folivoria como fonte suplementar de proteínas (ZORTÉA \& CHIARELLO 1994; BERNARD 1997), permitindo uma maior ocupação dos fundos de vales e praças.

Tabela II. Espécies coletadas em abrigos na região urbana de Londrina entre abril de 1998 e março de 1999 e tipos de abrigos utilizados.

\begin{tabular}{|c|c|c|c|c|c|c|c|c|c|c|c|c|c|c|c|}
\hline \multirow{2}{*}{ Espécies } & \multicolumn{14}{|c|}{ Abrigos utilizados ${ }^{*}$} & \multirow{2}{*}{$\begin{array}{l}\text { Total de } \\
\text { individuos }\end{array}$} \\
\hline & 1 & 2 & 3 & 4 & 5 & 6 & 7 & 8 & 9 & 10 & 11 & 12 & 13 & 14 & \\
\hline Noctilio leporinus & & & & & & & & & & & $x$ & & & & 8 \\
\hline Artibeus lituratus & & & & & & & & & & & & & $x$ & & 7 \\
\hline Carollia perspicillata & & $x$ & & & & & & & & & & & & & 25 \\
\hline Platyrninus lineatus & & & & & & & $x$ & & & & & & & & 1 \\
\hline Eptesicus brasiliensis & & & & & & & & & & & & & & $x$ & 14 \\
\hline Lasiurus borealis & & & & & & & & & & & & $x$ & & & 1 \\
\hline Lasiurus ega & & & & & & & & $x$ & & & & & & & 1 \\
\hline Eumops glaucinus & & & & & $x$ & & & & & & & & & & 20 \\
\hline Molossus rufus & $x$ & & $x$ & & $x$ & & & & & & & & & & 214 \\
\hline Molossus molossus & & & $x$ & & & & & & & $x$ & & & & & 38 \\
\hline Nyctinomops laticaudatus & & & & $x$ & & $x$ & & & & & & & & & 201 \\
\hline Nyctinomops macrotis & & & & & $x$ & & & & $x$ & & & & & & 47 \\
\hline Total de individuos por abrigo & 124 & 25 & 84 & 200 & 95 & 1 & 1 & 1 & 1 & 15 & 8 & 1 & 7 & 14 & 577 \\
\hline Total de abrigos utilizados & 4 & 2 & 6 & 1 & 4 & 1 & 1 & 1 & 1 & 1 & 1 & 1 & 1 & 1 & \\
\hline
\end{tabular}

(1) Forro de prédio, (2) tubulação fluvial no P.M. Arthur Thomas, (3) forro de casa/alvenaria, (4) pedreira abandonada, (5) junta de dilatação entre prédios, (6) apartamento no nono andar, (7) toldo em casa, (8) interior de uma churrasqueira de tijolos, (9) sacada de prédio, (10) forro de casa/madeira, (11) oco de árvore na margem do lago Igapó, (12) quintal de casa, (13) copa de árvore, (14) ar condicionado.

Quanto às espécies capturadas nesta pesquisa em edificações urbanas, $75 \%$ eram insetívoras $(25 \%$ pertenciam à família Vespertilionidae e $50 \%$ à família Molossidae), o que se deve, principalmente, ao tipo de metodologia utilizada na captura, feita nos abrigos durante as coletas diurnas; as frugívoras (Phyllostomidae) perfizeram $17 \%$ das coletas, e foram registradas em abrigos arbóreos de folhagem densa próximos às edificações, como sete-copas (Terminalia catappa L., Combretaceae) e coquinho (Syagrus romanzoffiana (Cham.) Glassm., Arecaceae); as piscívoras (Noctilionidae), $8 \%$ das espécies capturadas, foram encontradas nas margens do lago Igapó, que oferece condições de alimentação e abrigo em sua vegetação marginal (Tab. I). Essa alta incidência de espécies insetívoras, bem como a predominância da família Molossidae, está de acordo com os resultados encontrados por BREDT \& UIEDA (1996) no Distrito Federal. 
Quanto às coletas em edificações, estas requerem um esforço maior durante as capturas, pelo fato de os abrigos utilizados serem de difícil acesso ou apresentarem possíveis riscos para o coletor, como é o caso de juntas de dilatação entre prédios, fendas de pedras e forros de prédios. Nos fundos de vales e no campus da UEL, onde coletas noturnas foram feitas com uso de redes-neblina, as coletas mais expressivas foram das espécies da família Phyllostomidae, que perfizeram $47 \%$ do total aí coletado (Tab. I). Essa metodologia é, de certa forma, seletiva, pois as redes são armadas ao nível das frutas disponíveis, isto é, entre 0,5 a $2,5 \mathrm{~m}$ do solo, e isso facilita a captura de morcegos frugívoros; as espécies insetívoras, por usarem com maior freqüência a ecolocalização para caçar suas presas, detectam com facilidade as redes (PEDRO \& TADDEI 1997), e os molossídeos voam acima da copa das árvores (HANDLEY 1967).

Apesar da comprovação, em vários estudos, da presença do vírus da raiva em morcegos urbanos, deve-se ponderar sobre os incômodos e transtornos que esses animais causam comparando com os benefícios que trazem. Sabe-se que os morcegos insetívoros são responsáveis, em grande parte, pelo controle das populações de insetos e que muitas espécies de nectarívoros e frugívoros são potenciais agentes polinizadores e dispersores de sementes, o que é fundamental na recuperação e manutenção de áreas degradadas, como geralmente são os fundos de vales das áreas urbanas.

Campanhas de esclarecimento sobre os cuidados que devem ser tomados em relação a esses animais - não manuseá-los, evitando assim o risco de contrair raiva; usar máscara respiratória na retirada das fezes dos forros para evitar a inalação do fungo Histoplasma capsulatum (causador da histoplasmose), presente em suas fezes; procurar serviços especializados quando em contato com um morcego (se possível levando o animal); evitar que animais domésticos entrem em contato com eles -, além de medidas estratégicas de vacinação de animais domésticos e de rebanhos bovinos, aliadas a planejamentos arquitetônicos que levem em consideração a utilização de espaços residenciais como abrigo desses animais poderiam diminuir conflitos entre homens e morcegos.

\section{REFERÊNCIAS BIBLIOGRÁFICAS}

Almeida, M.A.; E.A.C. Aguiar; L.F.A. Matorelli \& M.M.S. Silva. 1994. Diagnóstico laboratorial de raiva em quirópteros realizado em área metropolitana na região sudeste do Brasil. Rev. Saúde Públ., São Paulo, 28 (5): 341-344.

BAER, G.M. 1991. Rabies in nonhematophagous bats, p. 341-366. In: G.M. BAER (Ed.). The natural history of rabies. Florida, CRC Press, 603p.

BERnARD, E. 1997. Folivory in Artibeus concolor (Chiroptera: Phyllostomidae): a new evidence. Chirop. Neotrop. 3 (2): 77-79.

Bredt, A.I.; F.A.A. ARaúJo; J. Caetano-JÚnior; M.G.R. Rodrigues; M. Yoshizawa; M.M.S. Silva; N.M.S. Harmani; P.N.T. Massunaga; S.P. BÜrer; V.A.R. Potro \& W. Uieda. 1996. Morcegos em áreas urbanas e rurais: manual de manejo e controle. Brasília, Fundação Nacional de Saúde, Ministério da Saúde, 117p.

BREDT, A. \& W. UiedA. 1996. Bats from urban and rural environments of the Distrito Federal, mid-western Brazil. Chirop. Neotrop. 2 (2): 54-57.

BRUMmITT, R.K. 1992. Vascular plant families and genera. Kew, Royal Botanic Gardens, 804p. 
Greenhall, A.M. \& J.L. Paradiso. 1968. Bats and bat banding. Washington, DC, Bureau of Sport Fisheries and Wildl, 47p.

HANDLEy JR., C.O. 1967. Bats of the canopy of an Amazonian Forest. Atas do Simpósio sobre a Biota Amazônica, Zool., 5: 211-215.

Husson, A.M. 1962. The bats of Suriname. Zool. Verh., Leiden, 58: 1-282.

IвGE. 2000. Sinopse preliminar do censo demográfico. Rio de Janeiro, Ministério de Planejamento, Orçamento e Gestão, Instituto Brasileiro de Geografia e Estatística, vol. 7, $3^{\mathrm{a}}$ parte, 100p.

JONES, J.K. \& D.C. CARTER. 1976. Annotated checklist with keys to subfamilies and genera. In: Biology of bats the New World family Phyllostomatidae. Part I. Spec. Publ. Mus. Texas Tech. Univ. 10 : 7-38.

PEDRo, W.A. 1998. Morcegos na área urbana. Biológico, São Paulo, 60 (2): 101-102

PEDRo, W.A. \& V.A. TADDEI. 1997. Taxonomic assemblage of bats from Panga Reserve, southeastern Brazil: abundance patterns and trophic relations in the Phyllostomidae (Chiroptera). Bol. Mus. Biol. Mello Leitão, Santa Teresa, 6: 3-21.

REIS, N.R. DOS \& M.F. MULLER. 1995. Bat diversity of forests and open areas in a subtropical region of south Brazil. Ecologia Austral, Córdoba, 5: 31-36.

Reis, N.R. Dos; M.F. Muller; E.S. Soares \& A.L. Peracchi. 1993a. Lista de quirópteros do Parque Estadual Mata do Godoy e arredores de Londrina-Paraná. Semina, Ci. Biol. Saúde, Londrina, 4 (2): 120-126.

Reis, N.R. Dos; A.L. Peracchi \& M.K. OnUki. 1993b. Quirópteros de Londrina, Paraná, Brasil (Mammalia, Chiroptera). Revta bras. Zool. 10 (3): 371-381

Reis, N.R. dos; A.L. Peracchi; I.P. De Lima; M.L. Sekiama \& V.J. Rocha. 1998. Updated list of the chiropterians of the city of Londrina, Paraná, Brazil. Chirop. Neotrop. 4 (2): 96-98.

Reis, N.R. dos; A.L. Peracchi; M.L. Sekiama \& I.P. DE Lima. 2000. Diversidade de morcegos (Chiroptera, Mammalia) em fragmentos florestais no estado do Paraná, Brasil. Revta bras. Zool. 17 (3): 697-704

RYDELL, J. 1992. Exploitation of insects around streetlamps by bats in Sweden. Functional Ecol. 6 : 744-750.

SEPLAN. Centro de pesquisas e Informações. 1993. Perfil de Londrina - Dados estatísticos. 1989-1992 Londrina, Seplan (Secretaria de Planejamento), 102p.

Silva, L.H.Q. Da; E.M.S. Cunha; W.A. Pedro; T.C. Cardoso; M. do C. DE C. Souza \& C.I.L. Ferrari 1999. Isolamento do vírus rábico em Molossus ater (Chiroptera: Molossidae) no estado de São Paulo. Rev. Saúde Públ., São Paulo, 33 (6): 626-628.

Silva, L.H.Q. DA; C.I.L. FerRari; Z.M.P. Peixoto; E.M.S. Cunha; C.M. GonZales. 1996a. Diagnóstico laboratorial da raiva na região de Araçatuba no período de janeiro/1993 a dezembro/1995. Biológico, São Paulo, 58: 7-12.

Silva, M.M.S.; N.M.S. Harmani; E.F.B. Gonçalves \& W. Uieda. 1996b. Bats from the metropolitan region of São Paulo, southeastern Brazil. Chirop. Neotrop. 2 (1): 39-41.

Uieda, W. 1998. Rabies in the insectivorous bat Tadarida brasiliensis in southeastern Brazil. Rev. Saúde Pública, São Paulo, 32 (5): 484-5.

UiEDA, W.; M.M. HAYASHI; L.H. Gomes \& M.M.S. SiLvA. 1996. Espécies de quirópteros diagnosticadas com raiva no Brasil. Bol. Inst. Pasteur, São Paulo, 1: 17-35

VIzoTTO, L.D.\& V.A. TADDEI. 1973. Chave para determinação de quirópteros brasileiros. Bol. Ciências, São Paulo, 1: 1-72.

ZortÉA, M. \& A.G. ChiARello. 1994. Observations on the big fruit-eating bat, Artibeus lituratus in an urban reserve of south east Brazil. Mammalia 58 (4): 665-670.

Recebido em 11.I.2002; aceito em 23.VII.2002. 